\title{
Leg Ulceration in Rheumatoid Arthritis - An Underreported Multicausal Complication with Considerable Morbidity: Analysis of Thirty-Six Patients and Review of the Literature
}

\author{
Cornelia S. Seitz $^{\mathrm{a}, \mathrm{b}} \quad$ Nikolaus Berens ${ }^{\mathrm{a}} \quad$ Eva-B. Bröcker ${ }^{\mathrm{a}} \quad$ Axel Trautmann $^{\mathrm{a}}$ \\ Departments of Dermatology, Venereology and Allergology, ${ }^{a}$ University of Würzburg, Würzburg, and \\ ${ }^{b}$ University of Göttingen, Göttingen, Germany
}

\section{Key Words}

Rheumatoid arthritis - Leg ulcer $\cdot$ Chronic venous insufficiency • Vasculitis - Inactivity

\footnotetext{
Abstract

Background: Rheumatoid arthritis (RA) is a systemic inflammatory disease which may present with extra-articular symptoms, including cutaneous manifestations. Ulcerated rheumatoid nodules, necrotic vasculitic lesions and pyoderma gangrenosum are fairly characteristic and well-recognized causes of skin ulcers in RA. However, most RA patients develop leg ulcers due to other pathophysiological factors posing a diagnostic and therapeutic challenge and leading to considerable morbidity. Methods: A retrospective chart analysis of all patients with RA and leg ulcers hospitalized at our Dermatology Department between January 1998 and March 2008 was performed to evaluate risk factors and identify underlying conditions that predispose RA patients to the development of leg ulcers. Results: A total of 36 patients with RA and leg ulcers were identified. Three patients presented with necrotizing vasculitis and 2 with pyoderma gangrenosum. Chronic venous insufficiency was diagnosed as the underlying cause of
}

leg ulcers in 8 patients, peripheral arterial disease in 4 patients, and combined arterial and venous malfunction in 3 patients. Five patients suffered from pressure ulcers. Interestingly, in 11 patients (31\%) other underlying causes besides constricted mobility followed by secondary lymphedema could not be identified, and these ulcers were classified as 'inactivity leg ulcers'. Conclusions: The majority of leg ulcers in patients with RA are due to underlying venous/arterial malfunction while vasculitic or traumatic ulcers are less common. Additionally, we identified a relevant subgroup of patients with 'inactivity ulcers' due to impaired mobility and consecutive lymphedema. Morphology and localization of ulcerations as well as duplex sonography provide the most important clues for accurate diagnosis, ensuring adequate treatment.

Copyright $\odot 2010$ S. Karger AG, Basel

\section{Introduction}

Rheumatoid arthritis (RA) is a chronic inflammatory disease affecting about $1 \%$ of the population. It is clinically characterized by joint inflammation, progressive joint destruction and decreasing function- al ability. Systemic involvement may present with extra-articular manifestation of various organs, including the lung, heart, kidney and skin [1]. Recent studies focusing on dermatological conditions in RA patients have identified a high prevalence of rheumatoid nodules (34\%) and Raynaud phenomenon (17\%) [2-5]. According to different studies, the frequency of leg ulceration in patients with RA may be up to $10 \%[6,7]$ compared to the prevalence of venous leg ulceration in the general population of approximately $1 \%$ [8]; thus, implying that patients with RA are at increased risk of developing ulcerations of the lower extremities leading to considerable morbidity.

In this study, a cohort of 36 RA patients with leg ulcers was retrospectively analyzed to identify underlying conditions and risk factors for development of chronic lower extremity ulcerations.

\section{Case Report}

We present a 69-year-old woman with a 7-month history of leg ulceration located on the lateral malleolus. The ulceration developed after incidental trauma

\section{KARGER}

Fax +4161306 1234 E-Mail karger@karger.ch www.karger.com
Cornelia S. Seitz, MD

Department of Dermatology, Venereology and Allergology, University of Göttingen von-Siebold-Strasse 3

DE-37075 Göttingen (Germany)

Tel. +49551 396 819, Fax +49551391 3521, E-Mail cseitz@med.uni-goettingen.de 
and slowly progressed. Upon presentation, the size of the ulceration was $3 \times 2$ $\mathrm{cm}$ in diameter and $2.2 \mathrm{~cm}$ in depth. The patient had suffered from RA for 15 years leading to considerably impaired mobility. RF was $14.7 \mathrm{U} / \mathrm{ml}$ (reference interval 0-14 U/ml). The current treatment regimen consisted of methotrexate $(15 \mathrm{mg}$ weekly), low-dose prednisolone (5 $\mathrm{mg}$ daily), leflunomide and etoricoxib. The leg ulceration was colonized with Pseudomonas aeruginosa and Gram-positive bacilli. Dysfunction of the deep and superficial venous system was excluded by color duplex ultrasonography. She had a normal ankle-brachial index and no peripheral arterial disease (PAD). Lymphedema of the lower leg could be observed. The ulceration was treated by shave excision of the ulcer margins (histological examination of the obtained specimen did not show vasculitic changes) and vacuum-assisted closure. The immunosuppressive therapy was retained unchanged. With this treatment regimen, the ulceration slowly healed.

\section{Methods}

\section{Patients}

Between January 1998 and March 2008, a total of 36 consecutive patients with RA fulfilling the criteria of the American College of Rheumatology [9] were treated as inpatients at the Department of Dermatology, University of Würzburg, with leg ulcerations. A retrospective chart analysis of RA patients was performed and the following data were recorded: duration of RA, treatment for RA, smoking habits and additional systemic diseases (e.g. diabetes mellitus, hypertension). Body mass index was calculated according to the height and weight of the individuals. RA patients with coexisting connective tissue diseases were excluded.

\section{Diagnostic Workup}

Leg ulcer duration, relapse rate, horizontal and vertical localization of the ongoing ulceration, number and size of ulcers, as well as unilateral or bilateral leg involvement were documented. Results of clinical exams were recorded, including venous arterial disease and PAD, occurrence of polyneuropathy and lymphedema, and degree of immobility (i.e. restricted ankle movement, difficulties walking or wheelchair-bound). Venous function was assessed by color duplex ultrasonography of reflux of the deep and superficial venous system using LOGIQTM 500 (GE Medical Systems, Milwaukee, Wisc., USA). The deep venous system was judged by compressibility of the veins, filling defects, recanalized intraluminal channels and wall thickening. Arterial examination included determination of the ankle-brachial index and extent of atherosclerotic plaques in the femoral artery by color duplex ultrasonography. Venous and arterial examinations were performed by the same physician (N.B.); only 3 patients had been examined by a different physician. Additional data in selected cases included results of histological analysis of perilesional biopsy, direct immunofluorescence and radiological examination to rule out vasculitis or underlying osteomyelitis, respectively.

\section{Laboratory Parameters}

Laboratory tests included complete blood count, rheumatoid factor, antinuclear antibody, erythrocyte sedimentation rate (ESR), C-reactive protein (CRP) and testing for bacterial colonization of the ulcers.

\section{Results}

\section{Patients}

The studied series consisted of 30 women and 6 men with a median age of 71 years (range 45-92 years). The majority of patients $(23 / 29,79 \%$; 7 patients with unknown duration) had suffered from RA for more than 10 years and 52\% (15/29) for more than 20 years. Thirty-five patients received various systemic treatments (table 1), including disease-modifying antirheumatic drugs, oral steroids, methotrexate, combination of methotrexate and oral steroids, or others including azathioprine $(\mathrm{n}=2)$, azulfidine $(\mathrm{n}=1)$, combination of azulfidine and hydroxychloroquine $(\mathrm{n}=$ 1), leflunomide $(n=1)$, mycophenolate mofetil $(n=1)$, etanercept $(n=1)$ and adalimumab $(n=2)$. One patient was without specific treatment. The average BMI was 27 (range 19-44). Eight patients (21\%) suffered from diabetes, 23 patients (64\%) had arterial hypertension.

Clinical Characteristics of Ulcers

The mean duration of the leg ulceration was 12 months (range 1-60); 28\% of patients had a history of recurrent leg ul-
Table 1. Clinical characteristics of the studied RA patients

\begin{tabular}{lc}
\hline Patients, $\mathrm{n}$ & 36 \\
Mean age, years (range) & $71(45-92)$ \\
Females/males & $30 / 6$ \\
Medication & \\
DMARDs & 6 \\
Oral steroids & 7 \\
Methotrexate & 7 \\
Methotrexate/oral steroids & 6 \\
Biologicals & 3 \\
Others & 6 \\
No treatment & 1 \\
Disease duration & \\
Up to 5 years & 2 \\
6-10 years & 4 \\
11-20 years & 8 \\
More than 20 years & 15 \\
Unknown & 7 \\
Mobility & \\
Normal & 2 \\
Restricted ankle movement & 9 \\
Crutches & 16 \\
Wheelchair & 7 \\
Unknown & 2 \\
\end{tabular}

DMARDs = Disease-modifying antirheumatic drugs.

Table 2. Size and localization of leg ulcerations in the studied RA patients

$\begin{array}{lr}\text { Number of ulcers } & \\ 1 & 17 \\ 2 & 8 \\ >2 & 11 \\ \text { General localization } & \\ \text { One leg } & 26 \\ \text { Both legs } & 10 \\ \text { Gaiter area } & 27 \\ \text { Foot } & 6 \\ \text { Multiple sites } & 3 \\ \text { Horizontal axis } & \\ \text { Medial } & 6 \\ \text { Anterolateral } & 17 \\ \text { Dorsal } & 2 \\ \text { Multiple locations } & 11 \\ \left.\text { Size (cm }{ }^{2}\right) & \\ <10 & 9 \\ 10-20 & 7 \\ 21-30 & 6 \\ 31-100 & 5 \\ >100 & 9\end{array}$

Number of ulcers

7

8

1

eneral localization

One leg

26

10

27

6

6

7

2

1

9

7

6

5

9 


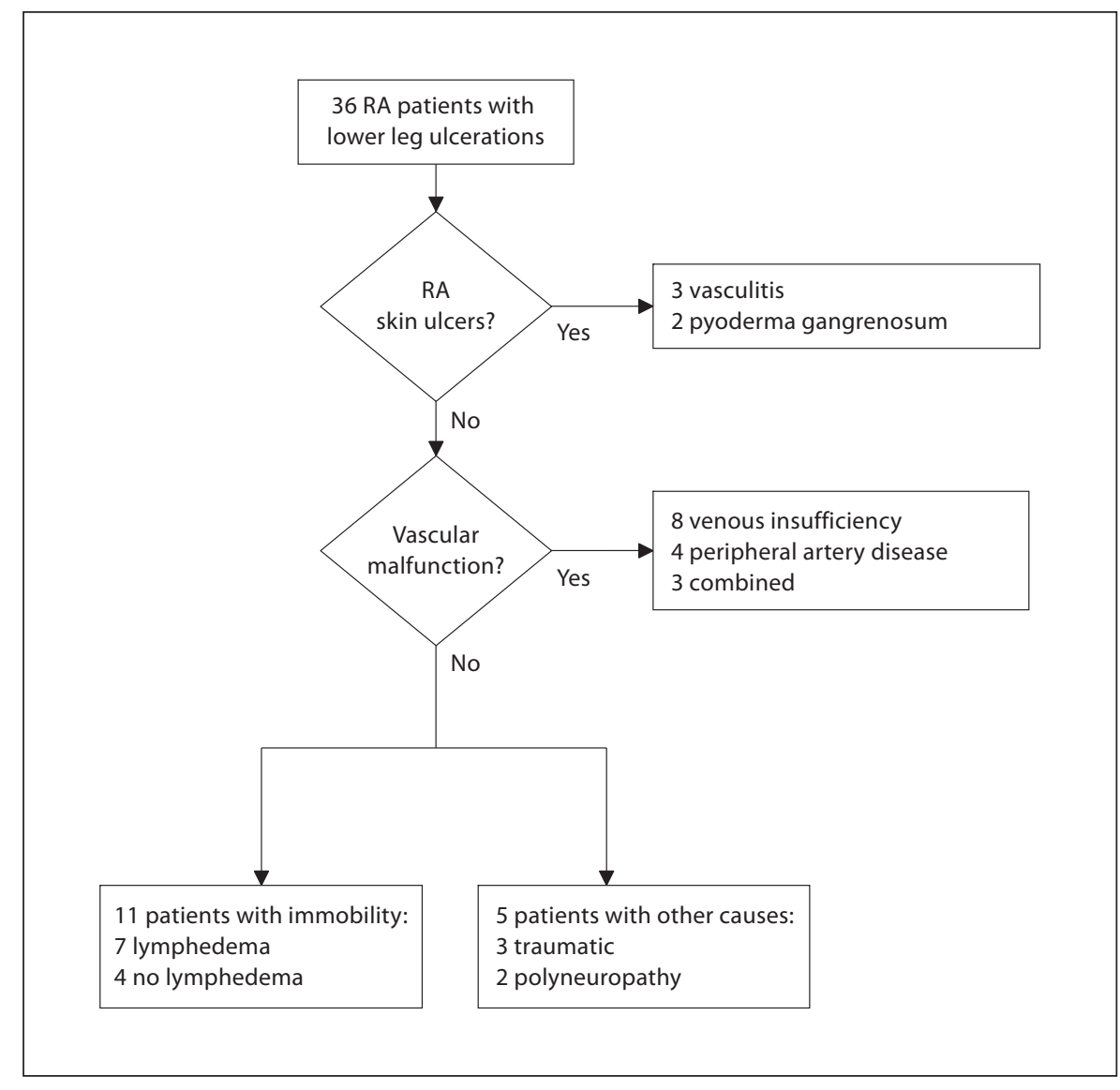

Fig. 1. Algorithm for classification of leg ulcers in RA.

ceration; $47 \%$ of patients had 1 ulcer, $22 \%$ had 2 ulcers, and $31 \%$ had $>2$ ulcers. Further ulcer characteristics are summarized in table 2 . The ulcerations were located on 1 leg in $72 \%$ of patients; in $28 \%$ of patients both legs were affected. Ulcers were located in the gaiter area in $75 \%$ of cases (according to classification by Callam et al. [10]), 17\% cases had ulcerations on the foot, and $8 \%$ of cases presented with ulcers at multiple locations. On the horizontal axis, ulcers were located medially in $17 \%$, anteriorly/laterally in $47 \%$, and dorsally in $6 \%$ of patients. In total, $30 \%$ of patients presented with ulcers at multiple sites. The size of the ulcer varied considerably as shown in table 2. Underlying osteomyelitis was excluded in 10 cases. Biopsies of ulcer margins were performed in 14 of 36 patients. In 3 patients, vasculitis was histologically evident, while 11 patients did not show vasculitic changes. The final diagnosis and classification of leg ulcers in our patients has been summarized in a flowchart (fig. 1).

\section{Immunological Ulcers}

Necrotizing vasculitis was identified by histology and direct immunofluorescence in 3 cases. In 11 patients, vasculitis was ruled out by biopsy of the ulcer margin. An additional 2 cases were diagnosed as pyoderma gangrenosum (fig. 2D).

\section{Vascular Malfunction}

Chronic venous insufficiency was identified in $33 \%$ of patients $(12 / 36)$, postthrombotic syndrome in $17 \%$ of patients $(6 / 36)$, and dysfunction of the superficial and deep venous system was found in $8 \%$ (3/36) (fig. 2A). Atherosclerotic plaques were identified by ultrasound in $52 \%$ of patients. Approximately $20 \%$ of patients (7/36) had relevant PAD (fig. 2C). Combined venous and arterial ulcer was diagnosed in 3 patients (8\%).
Traumatic Ulcers

In 3 patients (8\%), pressure ulcers due to deformities of the foot were identified. In 2 patients, ulcerations were caused by trauma and underlying polyneuropathy.

\section{Inactivity Ulcers}

A total of 11 patients (31\%) had neither a venous, arterial nor vasculitic disorder, nor any of the other previously mentioned underlying disorders. This group was classified as 'inactivity ulcer due to RA' (fig. 2B). In this subgroup, $64 \%$ of patients (7/11) had lymphedema and/or showed significantly impaired mobility. The inactivity ulcers' were located anteriorly/laterally in $36 \%$ (4/11, including the presented case report; fig. $2 \mathrm{~B})$, medially in $9 \%(1 / 11)$, posteriorly in $9 \%(1 / 11)$ and mixed in $46 \%$ (5/11) of cases. In this subgroup, $18 \%$ of patients $(2 / 11)$ had a history of recurrent leg ulceration. There was no significant difference concerning duration and clinical activity of RA, laboratory parameters, or size of the ulceration compared to the rest of the cohort.

\section{Laboratory Parameters}

Blood count showed normal values for thrombocytes in the majority of cases, thrombocytosis was found in 19\% (7/36) of patients. In total, $78 \%(28 / 36)$ of patients suffered from mild-to-moderate anemia $(10-11.5 \mathrm{~g} / \mathrm{dl}), 14 \%(5 / 36)$ had severe anemia $(<10 \mathrm{~g} / \mathrm{dl})$ with 1 patient requiring blood transfusion. CRP was moderately elevated (between 1 and $5 \mathrm{mg} / \mathrm{dl}$ ) in $58 \%$ $(21 / 36)$, in $17 \%(6 / 36)$ of patients CRP was $>5 \mathrm{mg} / \mathrm{dl}$. ESR was between 50 and 80 $\mathrm{mm} / \mathrm{h}$ in $58 \%(21 / 36)$ of patients, $11 \%$ $(4 / 36)$ had an ESR of $>100 \mathrm{~mm} / \mathrm{h}$. Furthermore, $61 \%$ of tested patients $(22 / 36)$ had a positive rheumatoid factor, $50 \%$ of them with a high titer. Positive antinuclear antibody results were found in $22 \%(8 / 36)$ of patients, predominantly with a low titer $(<1: 160)$. Ulcers were colonized with Staphylococcus aureus in $31 \%(11 / 36)$, Pseudomonas aeruginosa in 14\% (5/36), a combination of both in $14 \%(5 / 36)$, or other Gram-negative bacteria in $8 \%(3 / 36)$.

\section{Discussion}

Chronic ulceration of the lower extremity is a fairly common medical problem affecting approximately $1 \%$ of the general population. For patients with RA, the prevalence of leg ulceration has been esti- 
mated in several studies to be up to $10 \%$ [6], rising to $34 \%$ in patients with systemic rheumatoid vasculitis (RV) $[7,11]$. In the general population, ulcers due to venous dysfunction contribute to $70-90 \%$ of all cases; however, in patients with RA the underlying causes are oftentimes multifactorial. In our series of patients, vascular dysfunction and immobility accounted for the majority of cases, whereas RA-specific vasculitic ulcerations or pyoderma gangrenosum were rather rare. Our cohort consisted of patients admitted to the hospital because ambulatory treatments had failed in the past. Therefore, studied patients may have been affected more severely than the general RA population and bias including overestimation of average size of ulceration as well as several co-factors, such as inactivity or arterial dysfunction, cannot be excluded.

$\mathrm{RV}$ is a severe complication of RA with increased morbidity and mortality affecting between 0.7 and $7.9 \%$ of RA patients depending on the studied cohort (community-based versus hospitalized) [12-15]. In our study, we identified a relatively low number of patients with ulcers of vasculitic etiology (8\%) compared to $18 \%$ [16], $37 \%$ [17] and 50\% [18] of patients in comparable studies. Diagnosis of cutaneous vasculitis is usually established by skin biopsy. However, biopsies of ulcer margins may show secondary changes due to bacterial superinfection as well as ongoing inflammatory stimuli mimicking vasculitis $[19,20]$. Due to these confounding factors, margin biopsies of ulceration may have led to overestimation of vasculitic changes in previous studies. Patients with long-standing disease, severe joint erosions and deformities, presence of rheumatoid nodules, and with high levels of rheumatoid factor are at higher risk of developing RV [1]. Predilection sites for these types of ulcers are the lateral malleolus or the pretibial area. RV ulcers are characterized by pronounced painfulness and a punchedout appearance. The considerable morbidity of RV, however, depends on the involvement of peripheral nerves (mononeuritis multiplex or distal symmetric sensory neuropathy), as well as CNS, heart, lung, kidney, bowel and ocular involvement [21]. Treatment options for RV leg ulcerations include systemic steroids, methotrexate [22] and leukocytapheresis [23].

Pyoderma gangrenosum is clinically characterized by ulceration with necrotic undermined borders and a tendency for
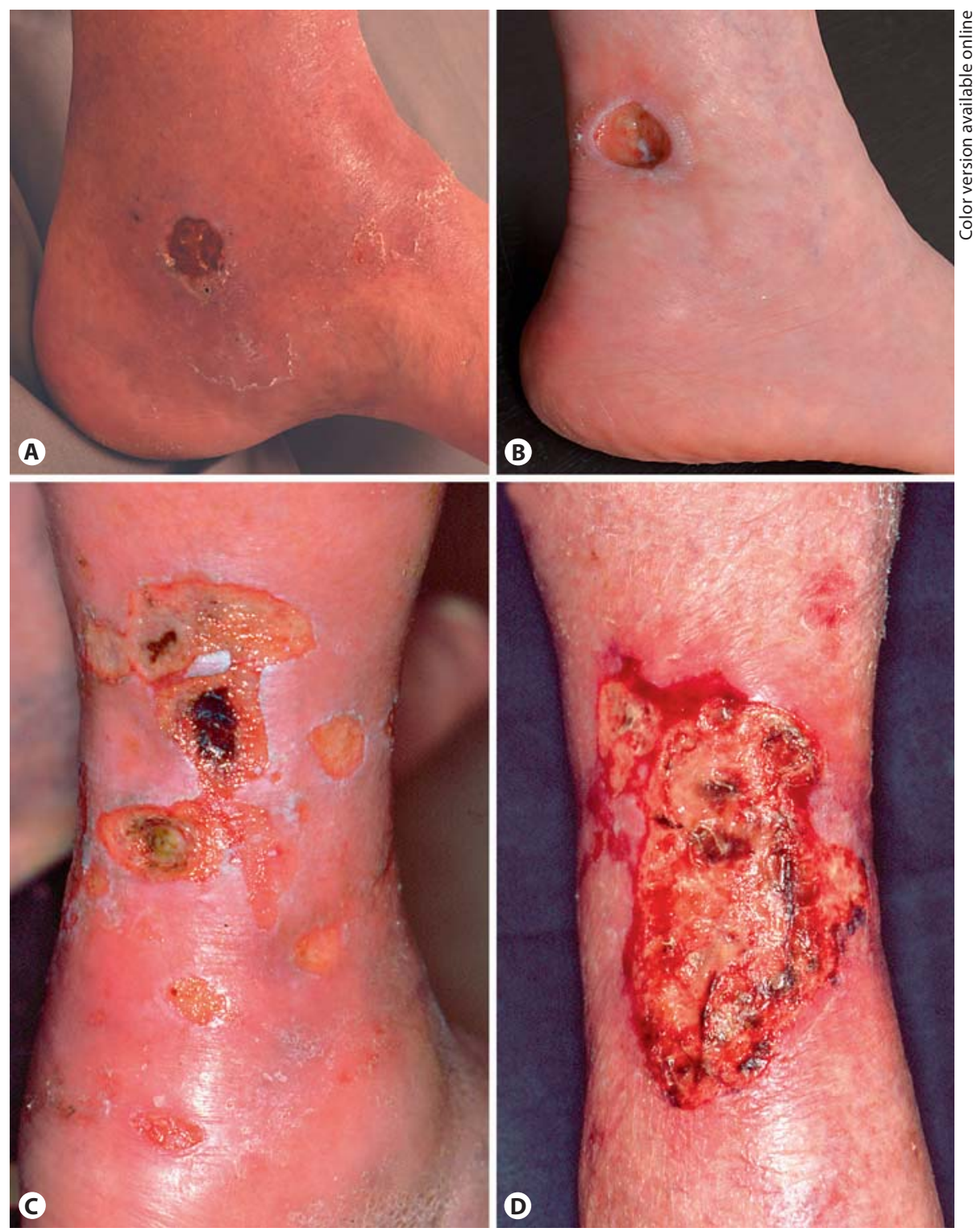

Fig. 2. Clinical appearance of leg ulcers in RA patients due to different underlying conditions. A Venous ulcer located at the medial malleolus surrounded by varicosis. Blue staining of the surrounding skin is due to gentian violet. B Deep 'inactivity ulceration' located on the lateral malleolus. C Coalescing ulcerations and black eschar necrosis on the dorsal aspect of the thigh due to PAD. D Pyoderma gangrenosum showing bizarre configuration of the ulceration rims, undermined edges, and a soft edematous ulcerated area.

rapid progression after minor trauma (fig. 2D). Due to these typical clinical features, we diagnosed pyoderma gangrenosum in 2 patients. Although pyoderma gangrenosum may be associated with RA, this type of skin ulceration may be found more often in patients with chronic inflammatory bowel disease and paraproteinemia [4].
In our study, insufficiency of the superficial and deep venous systems was identified as the main cause in $22 \%$ patients $(12 / 36), 3$ of these suffered from a combination of venous and arterial malfunction. Pun et al. [16] identified venous dysfunction as the most common cause of leg ulceration, accounting for $45 \%$ in a cohort of 
patients with underlying RA. The predilection sites of venous ulcers in RA patients correspond to those of venous ulcers in the general population: solitary or bilateral shallow ulcerations with a fibrinous base and irregular margins on the medial aspect of the lower leg, just above the malleolus, accompanied by typical cutaneous changes such as hemosiderin pigmentation, dermatosclerosis, atrophie blanche or obvious varicosis (fig. 2A) [8]. Rarely, venous ulcers are found laterally or proximally. In the majority of cases, aggravation of venous ulceration and impaired healing is due to concomitant impairment of the venous pump due to dysfunction of the ankle joint [24]. Accordingly, in our study $82 \%$ of patients with ulcers due to impaired venous function showed various degrees of constricted mobility. Standard treatment of patients with venous ulcers in our department includes shaving of the sclerotic areas/fasciotomy and subsequent vacuum-assisted split skin grafting. Concomitantly, a peri- and postoperative increase in immunosuppressive therapy by increasing the basic prednisolone dosage to $1 \mathrm{mg} / \mathrm{kg}$ body weight followed by slow tapering of the dose over a period of 3 weeks. An alternative treatment option of minor and painful ulcerations may be pinch grafting [18]; however, larger prospective studies are mandatory to identify optimal treatment options of venous ulcers in patients with RA.

RA patients tend to have an increased risk of developing atherosclerosis due to the disease itself and the long-standing immunosuppressive therapy with corticosteroids leading to increased morbidity by coronary, cerebral and PAD $[25,26]$. Relevant PAD is considered in patients with an ankle-brachial index $<0.9$ [27]. In our study, we identified 4 patients with PADinduced ulcers. Additionally, 3 patients suffered from combined arterial/venous ulcers. Typical clinical features of PAD include intermittent claudication induced by exercise, and relieved after $10 \mathrm{~min}$ of rest. However, many RA patients with movement restrictions due to their bone deformities may not experience these exercise-induced symptoms. Progression of PAD with ischemic rest pain may eventually lead to stage IV of PAD (according to classification of PAD by Fontaine and Rutherford), with gangrene affecting the digits, distal part of the forefoot and heel. In our patients, we observed arterial ulcerations of the leg predominantly on the anterior and lateral aspect of the lower leg (fig. 2C).

Deformities of the foot also represent a predisposition for skin ulcerations due to pressure, friction and minor trauma usually induced by ill-fitting shoes, e.g. flexion deformities of the toes predispose people to ulcerations on the dorsum of the toes [19]. A recent study by Firth et al. [28] suggests that the overall prevalence of foot ulcerations in patients with RA may be up to approximately $10 \%$. In this study, ulcers were most commonly found at the dorsal aspect of the hammer toes (48\%). Other critical areas of the foot are the plantar region around the metatarsal heads (32\%) (subluxation of metatarsophalangeal joints), the lateral and medial aspects of the forefoot adjacent to the metatarsophalangeal joint (20\%), and the heel [28]. In addition to increased skin fragility, polyneuropathy may also contribute to lesions of the foot. Therefore, podiatry care in patients with long-standing RA should be reserved to specialists, and personalized wide-fitting and extra-depth footwear is mandatory to prevent secondary ulcerations.

Infrequently, onset of leg ulcerations may develop during treatment with antiproliferative drugs [29]. In this context, development of vasculitis changes [30] and skin ulcerations $[31,32]$ has been observed in a few RA patients treated with the pyrimidine-synthesis inhibitor leflunomide, suggesting that leflunomide may play a role in the pathogenesis of a specific subset of leg ulcerations.

In 11 RA patients with leg ulcers, no underlying vascular or immunological condition could be identified. The majority of these patients including the presented case had restricted ankle movement, were immobile or wheelchair dependent and concomitantly displayed clinical signs of moderate-to-severe lymphedema of the leg (63\%) suggesting a secondary lymphedema due to constricted mobility. This group was termed 'inactivity ulcer due to RA' (fig. 2B). Interestingly, lymphedema predominantly affecting the upper extremities has been sporadically reported in long-standing RA [33]. However, a few cases of exclusive lower limb involvement have also been reported [34]. There is usually no correlation of lymphedema with seropositivity for rheumatoid factor or disease activity [34, 35]. The cause of edema in RA is currently unknown; however, immobility, inflammatory exudates around affected joints, hypo- proteinemia, increased capillary permeability and filtration as well as pre-existing abnormalities of the lymphatic vessels have been discussed in this context [36, 37]. Future detailed studies of the lymphatic system including lymphoscintigraphy in patients with RA and leg ulcerations may identify pathophysiological mechanisms in these patients. Clinically, the subgroup of patients with inactivity ulcers did not significantly differ from the remainder of RA patients. Our data show that peripheral edema may have been an underestimated cause of leg ulcers in RA in the past, and all efforts should be taken to minimize lymphedema in patients with RA by elevation of the affected extremity and application of adequate compression bandages [38].

Lower leg ulcerations in RA patients are oftentimes resistant to conventional therapies, and therefore pose a diagnostic and therapeutic challenge for the treating rheumatologist. Morphology, localization, time course, co-morbidity and clinical symptoms of the ulcerations give important hints for the exact diagnosis ensuring adequate treatment. Beside vascular malfunction, in our study we identified a relevant subgroup of patients with 'inactivity ulcers' presenting with mild-to-moderate lymphedema and reduced mobility. It is tempting to speculate that lymphedema is a consequence of impaired mobility. However, whether lymphedema in individual patients with RA and leg ulcerations is primary or secondary in nature has to be addressed by future studies including lymphoscintigraphy.

\section{References}

1 Turesson C, Matteson EL: Management of extra-articular disease manifestations in rheumatoid arthritis. Curr Opin Rheumatol 2004;16:206-211.

2 Hata T, Kavanaugh A: Rheumatoid arthritis in dermatology. Clin Dermatol 2006;24: 430-437.

3 Douglas KM, Ladoyanni E, Treharne GJ, Hale ED, Erb N, Kitas GD: Cutaneous abnormalities in rheumatoid arthritis compared with non-inflammatory rheumatic conditions. Ann Rheum Dis 2006;65:1341-1345.

4 Sayah A, English JC 3rd: Rheumatoid arthritis: a review of the cutaneous manifestations. J Am Acad Dermatol 2005;53:191-209.

5 Jorizzo JL, Daniels JC: Dermatologic conditions reported in patients with rheumatoid arthritis. J Am Acad Dermatol 1983;8:439457. 
6 Thurtle OA, Cawley MI: The frequency of leg ulceration in rheumatoid arthritis: a survey. J Rheumatol 1983;10:507-509.

7 McRorie ER, Jobanputra P, Ruckley CV, Nuki G: Leg ulceration in rheumatoid arthritis. Br J Rheumatol 1994;33:1078-1084.

$\checkmark 8$ Valencia IC, Falabella A, Kirsner RS, Eaglstein WH: Chronic venous insufficiency and venous leg ulceration. J Am Acad Dermatol 2001;44:401-421.

-9 Arnett FC, Edworthy SM, Bloch DA, et al: The American Rheumatism Association 1987 revised criteria for the classification of rheumatoid arthritis. Arthritis Rheum 1988; 31:315-324.

10 Callam MJ, Harper DR, Dale JJ, Ruckley CV: Chronic ulcer of the leg: clinical history. $\mathrm{Br}$ Med J 1987;294:1389-1391.

-11 Scott DG, Bacon PA: Intravenous cyclophos phamide plus methylprednisolone in treatment of systemic rheumatoid vasculitis. Am J Med 1984;76:377-384.

12 Salvarani C, Macchioni P, Mantovani W, Rossi F, Veneziani M, Boiardi L, Lodi L, Portioli I: Extraarticular manifestations of rheumatoid arthritis and HLA antigens in northern Italy. J Rheumatol 1992;19:242-246.

-13 Kaye O, Beckers CC, Paquet P, Arrese JE, Pierard GE, Malaise MG: The frequency of cutaneous vasculitis is not increased in patients with rheumatoid arthritis treated with methotrexate. J Rheumatol 1996;23:253-257.

14 Turesson C, Jacobsson L, Bergstrom U: Extra-articular rheumatoid arthritis: prevalence and mortality. Rheumatology (Oxford) 1999;38:668-674.

15 Chen KR, Toyohara A, Suzuki A, Miyakawa S: Clinical and histopathological spectrum of cutaneous vasculitis in rheumatoid arthritis. Br J Dermatol 2002;147:905-913.

16 Pun YL, Barraclough DR, Muirden KD: Leg ulcers in rheumatoid arthritis. Med J Aust 1990;153:585-587.
17 Wilkinson M, Kirk J: Leg ulcers complicating rheumatoid arthritis. Scott Med J 1965; 10:175-182.

18 Oien RF, Hakansson A, Hansen BU: Leg ulcers in patients with rheumatoid arthritis-a prospective study of aetiology, wound healing and pain reduction after pinch grafting. Rheumatology (Oxford) 2001;40:816-820.

19 Cawley MI: Vasculitis and ulceration in rheumatic diseases of the foot. Baillieres Clin Rheumatol 1987;1:315-333.

20 Pierard-Franchimont C, Paquet P, Arrese JE, Pierard GE: Healing rate and bacterial necrotizing vasculitis in venous leg ulcers. Dermatology 1997;194:383-387.

21 Genta MS, Genta RM, Gabay C: Systemic rheumatoid vasculitis: a review. Semin Arthritis Rheum 2006;36:88-98.

22 Espinoza LR, Espinoza CG, Vasey FB, Germain BF: Oral methotrexate therapy for chronic rheumatoid arthritis ulcerations. J Am Acad Dermatol 1986;15:508-512.

23 Itoh Y, Takeshita Y, Ozawa Y, Tohma S, Umemura S: A case report of leukocytapheresis for refractory leg ulcers complicated with rheumatoid arthritis. Ther Apher Dial 2006;10:419-424.

24 McRorie ER, Ruckley CV, Nuki G: The relevance of large-vessel vascular disease and restricted ankle movement to the aetiology of leg ulceration in rheumatoid arthritis. Br J Rheumatol 1998:37:1295-1298.

25 del Rincon I, Escalante A: Atherosclerotic cardiovascular disease in rheumatoid arthritis. Curr Rheumatol Rep 2003;5:278-286.

26 Hahn BH, Grossman J, Chen W, McMahon $\mathrm{M}$ : The pathogenesis of atherosclerosis in autoimmune rheumatic diseases: roles of inflammation and dyslipidemia. J Autoimmun 2007;28:69-75.

27 Norgren L, Hiatt WR, Dormandy JA, Nehler MR, Harris KA, Fowkes FG, Group TIW: Inter-Society Consensus for the Management of Peripheral Arterial Disease (TASC II). J Vasc Surg 2007;45(suppl):S5-S67.
28 Firth J, Hale C, Helliwell P, Hill J, Nelson EA: The prevalence of foot ulceration in patients with rheumatoid arthritis. Arthritis Rheum 2008;59:200-205.

29 Sirieix ME, Debure C, Baudot N, Dubertret L, Roux ME, Morel P, et al: Leg ulcers and hydroxyurea: forty-one cases. Arch Dermatolol 1999;135:818-820.

30 Holm EA, Balslev E, Jemec GBE: Vasculitis occurring during leflunomide therapy. Dermatology 2001;203:258-259.

31 McCoy CM: Leflunomide-associated skin ulceration. Ann Pharmacother 2002;36: 1009-1011.

-32 Knab J, Goos M, Dissemond J: Successful treatment of a leg ulcer occurring in a rheumatoid arthritis patient under leflunomide therapy. J Eur Acad Dermatol Venereol 2005; 19:243-246.

33 de Silva RT, Grennan DM, Palmer DG: Lymphatic obstruction in rheumatoid arthritis: a cause for upper limb oedema. Ann Rheum Dis 1980;39:260-265.

-34 Dacre JE, Scott DL, Huskisson EC: Lymphoedema of the limbs as an extra-articular feature of rheumatoid arthritis. Ann Rheum Dis 1990;49:722-724.

35 Grillet B, Dequeker J: Rheumatoid lymphedema. J Rheumatol 1987;14:1095-1097.

36 McRorie ER, Reid DM, Dunn NA, Nuki G: Lymphoedema associated with active inflammatory arthritis in a patient with congenital lymphatic hypoplasia. Br J Rheumatol 1995;34:689-691.

- 37 Tiwari A, Cheng KS, Button M, Myint F, Hamilton G: Differential diagnosis, investigation, and current treatment of lower limb lymphedema. Arch Surg 2003;138:152-161.

38 Prasad A, Ali-Khan A, Mortimer P: Leg ulcers and odema: a study exploring the prevalence, aetiology, and possible significance of oedema in venous ulcers. Phlebology 1990;5: 181-187. 